http://jmscr.igmpublication.org/home/ ISSN (e)-2347-176x ISSN (p) 2455-0450

crossref DOI: https://dx.doi.org/10.18535/jmscr/v9i1.36

\title{
Role of Diagnostics in Management of Breast Infection - A Retrospective Study from North Goa District Hospital
}

Authors

\section{Dr Shailendra Munj ${ }^{1}$, Dr Patricia Fernandes ${ }^{1}$, Dr Varsha Munj ${ }^{2}$, Dr Krupa Jog ${ }^{3}$, Dr Shruti Shetye}

Department of Surgery, Department of Pathology and Department of Microbiology North District Hospital

*Corresponding Author

Dr Shailendra Munj

Senior Surgeon, Surgery Department, North District Hospital, Peddem, Mapusa, Goa, Pin 403507, India

\author{
Abstract \\ Objective: To evaluate the utility of cytopathology and microbiological testing in diagnosis of breast \\ infections.
}

Materials \& Methods: This a retrospective study of cases presenting as mastitis and a review of 109 cases with regards to various laboratory tests in classification of mastitis and to identify their etiological agents.

Observation: Lactation was the risk factor for causing inflammatory breast abscess. Diabetes mellitus and tuberculosis also led to inflammatory breast abscess. 109 cases were included in this study. They were categorized into simple mastitis without abscess formation (24.7\%), acute mastitis (52.2\%), granulomatous mastitis (10\%), fat necrosis (6.4\%), hematoma (3.6\%) and mastitis not otherwise specified (2.7\%). Lactation was the risk factor in $44 \%$ cases of breast abscess. Diabetes mellitus and tuberculosis were responsible for non lactational inflammatory breast lesion. Granulomatous mastitis can be sub categorized by utilizing CNAAT, AFB staining \& TB culture. Fungal stain and fungal culture sensitivity helps in making a diagnosis of Fungal mastitis.

Conclusion: Laboratory diagnostics in the form of FNAC, microbiological testing and biochemical parameters can be utilized in the diagnosis of various breast infections for specific management of patients.

Keywords: Breast abscess, FNAC, granulomatous mastitis, Tuberculous mastitis, Fungal mastitis, Diabetes.

\section{Introduction}

Mastitis is a common complaint in clinical practice. Mastitis is defined as inflammation of breast with or without infection. ${ }^{1}$ Although significant number of cases are seen in lactating women, it can affect at any time. ${ }^{1,2}$ Mastitis with infection may be lactational or non lactational ${ }^{3}$. Breast abscess is localized collection of pus which can be a complication of mastitis causing great agony to women. ${ }^{4}$ Non infectious Mastitis includes idiopathic granulomatous mastitis, duct ectasias, and other causes like fat necrosis, hematoma etc. Mastitis does not raise the risk of breast cancer. But, uncommon type of breast cancer known as inflammatory breast cancer presents clinically like mastitis which can be mistaken for infection and cause a problem for diagnosis. ${ }^{5}$ Histopathology remains gold standard 
for establishing correct diagnosis, however Fine Needle Aspiration Cytology (FNAC) has gained significant credibility in initial diagnosis of breast diseases as it is simple,cost effective and quick OPD based test. When done by cytopathologist it can help in accurate and early diagnosis of this lesions. ${ }^{6}$ Breast aspirate should be handled systematically to get maximum information. Cytology combined with microbiological culture and other special studies can help in appropriate assessment of the lesion which will guide the clinician to administer appropriate therapy and decide on further course of treatment and also avoid unnecessary surgery. ${ }^{7}$

\section{Aim \& Objective}

1. To evaluate utility of diagnostics like cytopathology and microbiological test in diagnosis and management of breast infection.

2. To study the spectrum of inflammatory breast lesions.

3. To study the spectrum of microbiological pathogens leading to mastitis.

\section{Materials and Methods}

This is a retrospective study carried out in the Department of Pathology and Microbiology in coordination with Surgery Department. Cases were retrieved from department of hospital records, cytopathology and microbiology laboratory.

Total of 109 cases of Mastitis were seen in our hospital from January 2017 to January 2019. Clinical details like gender, age, lactational history, past history of $\mathrm{TB}$ and radiological findings if available were recorded. Routine
Hematological findings and biochemistry parameters like blood sugar was correlated. In all cases of mastitis presenting with breast lump cytological parameters were evaluated including presence of inflammatory cells, granulomas, histiocytes, giant cells and necrosis. All cases of pus and pus like aspirate were subjected to culture sensitivity, CNAAT testing in addition to AFB, PAS/GMS staining in microbiology section. The cytomorphologic features for acute mastitis included presence of numerous neutrophils, macrophages and benign ductal cells. Whenever histopathology was available the diagnosis was confirmed by $\mathrm{H}$ and $\mathrm{E}$ stained section and special stains like ZN Stain, PAS and GMS stain was applied.

The patients were classified into granulomatous mastitis based on presence of epitheloid granulomas, caseous necrosis and inflammatory cells. They were further classified into infectious and non infectious based on microbiology findings. 2 cases of duct carcinoma presenting with clinical features of mastitis were excluded from the study. 27 patients presented with simple mastitis without any lump. They were managed with hot fomentation and antibiotics and no FNAC was done in these cases.

\section{Results}

Total of 109 cases were diagnosed with mastitis clinically. All the patients were females. 27 cases were managed with analgesic and antibiotics based on clinical severity. 82 cases who presented with breast lump/ in duration were subjected to FNAC and microbiological studies. Results are displayed in Table 1 and Table 2.

Table 1: Classification of Mastitis

\begin{tabular}{|l|c|c|c|c|}
\hline Type of mastitis & Lactational & Non lactational & Inflammatory & Non inflammatory \\
\hline Simple mastitis & 17 & 10 & - & - \\
\hline Acute mastitis/abscess & 30 & 27 & 35 & 22 \\
\hline Granulomatous mastitis & 1 & 10 & 3 & 8 \\
\hline Fat necrosis & - & 7 & 1 & 6 \\
\hline Hematoma & - & 4 & 1 & 3 \\
\hline Mastitis not otherwise specified & - & 3 & - & 3 \\
\hline Total & 48 & 61 & 40 & 42 \\
\hline
\end{tabular}


Table 2: Distribution of microbiological isolates

\begin{tabular}{|l|c|}
\hline Organism & Number of cases \\
\hline $\begin{array}{l}\text { 1.Negative culture and } \\
\text { microscopy }\end{array}$ & 42 \\
\hline $\begin{array}{l}\text { 2.Fungal: } \\
\text { Candida Albicans }\end{array}$ & 01 \\
\hline 3.Tuberculosis & \\
TB & 02 \\
Atypical mycobacterium & 01 \\
\hline 4.Bacterial: & 26 \\
Staphylococcus aureus & 02 \\
Streptococus & 02 \\
Enterobactericeae & 03 \\
Corynebacterium & 02 \\
Escherichia Coli & 01 \\
Pseudomonas & \\
\hline
\end{tabular}

Table 3: Case Distribution of Inflammatory Breast Disease based on Blood sugar levels

\begin{tabular}{|l|c|c|}
\hline & Lactating & Non Lactating \\
\hline Increased FBSL/PPBSL & $2(1.8 \%)$ & $8(7.3 \%)$ \\
\hline Normal sugar levels & 46 & 53 \\
\hline
\end{tabular}

All the cases were seen in females. 59 cases were seen in left breast, 48 cases were in right breast and 2 cases in bilateral breasts. Mean age of presentation with mastitis was 32years, range (2265years).Mean age of presentation for lactating women was 23years, range (22-34years).Mean age of presentation for non lactating women was 34 years, range(26-65years).

Out of 12 cases of granulomatous mastitis diagnosed, 3 cases were diagnosed as Tuberculous based on cytology along with AFB, CNAAT and TB cultures. 2cases were CNAAT and AFB positive. 1case was AFB positive and CNAAT negative. Repeat FNAC was sent for TB culture which isolated atypical mycobacterium. Rest of the cases were diagnosed as idiopathic granulomatous mastitis after negative microbiology findings. 1 case of Candida was seen. Patient was a case of uncontrolled Diabetes Mellitus.

Data of blood sugar was available for all 109 cases from patient records. Refer table no. 3. Acute mastitis was managed with antibiotics and surgical drainage was done if they were not responsive to medical management. Subareolar abscess was treated by excision of abscess and lactiferous duct in addition to antibiotics. TB mastitis was started with anti tubercular drugs. In case of Fungal mastitis diabetic control, antibiotics, antifungal and surgical drainage was administered.

Idiopathic granulomatous mastitis treatment was challenging. 8 cases were started on steroids. Variable response to treatment was seen. A combination of antibiotics and surgery was used in treatment of these cases.

Histopathological evaluation was done in all cases where surgical intervention was done. FNAC correlated with histopathology in most of the cases. However, 2 cases of acute mastitis (abscess) with acute inflammatory reaction on FNAC were diagnosed as idiopathic granulomatous mastitis on histopathological evaluation.

In case of Fungal mastitis diabetic control, antibiotics, antifungal and surgical drainage was administered.

Duct ectasia, foreign body granuloma were managed surgically. No surgical intervention was done in case of fat necrosis and hematoma. They were managed clinically

\section{Discussion}

Benign breast disease is the most common cause of breast problems. $30 \%$ of women require treatment at some time in their lives. The most 
common symptoms are pain and lump in the breast. $^{8}$

Bacterial mastitis is the commonest variety of mastitis and commences acutely. It is most commonly associated with lactation in majority of the cases but it may not necessarily be so. ${ }^{89}$ In our study we found that infective mastitis was common in lactating women and non infective mastitis was common in non lactating women. Some of the cases of mastitis are associated with infected hematoma and with periductal mastitis.

In lactational mastitis most cases were caused by Staphylococcus aureus. The infants are usually the intermediary. After $2^{\text {nd }}$ day of life $50 \%$ infants harbor staphylococcus in the nasopharynx. Cleaning the baby's mouth with swab is an etiological factor. The mucosa is excoriated and becomes infected. The organism in infants saliva is inoculated on mother's nipple. Women with sore or cracked nipple are prone for ascending infection. Lactiferous duct gets blocked by epithelial debris leading to stasis of staphylococcus aureus causing clotting of milk and within this clot the organism multiplies. ${ }^{10,11}$

Diabetes mellitus has been associated with increased rates of infection. ${ }^{12,13,14}$ We found 9.1\% of women having Diabetes, $1.8 \%$ were lactational and $7.3 \%$ were non lactational. However the data available is limited and this needs a larger study to evaluate the findings for correlating the effect of Diabetes in causing mastitis. Mastitis was common in left breast than right breast. However, there is no comparative data available to determine predisposing factor and it needs a large scale study to understand the same. However, in our study we found that improper practice of breast feeding on Left side caused the same.

FNAC is considered a useful tool in the diagnosis of breast mass and lesions. It is the least invasive technique of obtaining a cell diagnosis and is very accurate if done by experienced cytologist. ${ }^{15,16}$ It differentiates malignant from benign cases and can be used in directing the management and treatment. FNAC aspirate when combined with microbiological cultures and specialized tests like
ZN staining, CBNAAT testing, PAS and GMS can further help in specific diagnosis from a single aspirate and enhance the management of these patients in the era of evidence based medicine ${ }^{.16}$ However a report of negative culture does not exclude infection as most cases receive antibiotic treatment quite early at the start of mastitis. ${ }^{17}$ This study highlights the use of ZN staining, CBNAAT in differentiating tuberculous mastitis from idiopathic granulomatous mastitis. Utilizing TB culture further enhances identification of atypical mycobacterium induced tuberculous mastitis. ${ }^{18}$ Risk factors for mastitis are primarily sore and cracked nipples, increased maternal age, obesity and tobacco smoking. ${ }^{19}$ In our study Diabetes Mellitus, extremes of age, wrong breast feeding practices and tuberculosis were risk factors for mastitis. Breast abscess was seen in pregnant and lactating women. Hence they need to be trained and counselled about breast feeding and hygiene of breast and nipples. ${ }^{12}$

Breast tuberculosis is a rare entity in the West as breast is relatively resistant to tuberculosis. However in India it constitutes 3\% of treatable breast lesions. ${ }^{20}$ Infection usually occurs as an extension of infection through contiguous structures such as chest wall, Tubercular pleurisy or through skin abrasions. ${ }^{21}$ FNAC showed epitheloid grnulomas with giant cells, caseous necrosis and neutrophils. One case presented like acute mastitis. In our study one patient was a known case of pulmonary TB. One of the cases had axillary and cervical lymphadenopathy. FNAC done thus proved it to be tuberculous lymphadenitis. One case had no history of TB or TB contact.

In our study idiopathic granulomatous mastitis was diagnosed by excluding infectious etiologies. These patients showed good response to steroid therapy. However some needed surgical intervention. They were difficult to treat as they came with recurrence and repeated surgical excisions were done in 2 cases. Idiopathic granulomatous mastitis showed non caseating granulomas with giant cells and few neutrophils. ${ }^{20}$ 
Candida albicans was isolated in a non lactating female with uncontrolled hyperglycemia. FNAC revealed neutrophils with histiocytes and reactive ductal cells with budding yeasts seen on smear. The aspirate submitted for PAS, GMS and fungal culture confirmed the diagnosis. ${ }^{15}$

Fat necrosis may be acute or chronic and occurs in stout middle aged women following blow or indirect violence (contraction of the pectoralis major). It mimics a carcinoma with skin tethering and nipple retraction. ${ }^{22}$ Triple assessment of breast was done in these cases, 4 cases showed resolution of lump. Biopsy confirmed fat necrosis. FNAC had a good correlation in diagnosis of fat necrosis. But based on radiological, clinical findings and presentation, biopsy can be done to rule out malignancy as fat necrosis may be seen with carcinoma of breast.

Cytology shows numerous foamy macropahges, giant cells and neutrophils in acute stage. In later stages lymphocytes and myofibroblastic cells areseen. Epithelial cells may appear atypical. Cultures are negative. ${ }^{23}$

\section{Conclusion}

It is evident from our study that breast infection (abscess) or infective mastitis is common in lactating women, however, it can occur at any age. Hence, stress has to be given on awareness of proper breast feeding and breast hygiene with special emphasis on infection control practices in nursery and maternity wards to prevent breast abscess formation. FNAC was found to be a useful tool in the diagnosis of infectious breast lesions and specific diagnosis can be done if pathologist submits the aspirate for microbiology studies. Tuberculosis should be considered a differential diagnosis for granulomatous mastitis in our area. Tuberculous mastitis should be differentiated from idiopathic granulomatous mastitis as treatment is completely different. Awareness of Breast care should emphasized in women with diabetes for prevention of breast abscess.

\section{References}

1. Verghese, B.G., Ravikanth, R. Breast Abscess, an Early Indicator for Diabetes Mellitus in Non-lactating Women: A Retrospective Study from Rural India. World J Surg 36, 1195-1198 (2012).

2. Richard J. Santen, and Robert Mansel. Benign Breast Disorders. N Engl J Med 2005; 353:275-285.

3. Rizzo M; Peng L; Frisch A; Jurado M; Umpierrez G. Breast abscesses in nonlactating women with diabetes: clinical features and outcome. Am J Med Sci. 2009; 338(2):123-6

4. Ramazan Eryilmaz, Mustafa Sahin, M. Hakan Tekelioglu, Emin Daldal. Management of lactational breast abscesses. The Breast 2005; volume 14, issue 5, P375-379.

5. B. Lonnerdal, "Nutritional and Physiologic Significance of Human Milk Proteins," The American Journal of Clinical Nutrition, 2003; Vol. 77, No. 6.

6. A.C. Thomsen,. T.Espersen,. S.Maigaard, Course and treatment of milk stasis, noninfectious inflammation of the breast, and infectious mastitis in nursing women, American Journal of Obstetrics and Gynecology, 1984; .Volume 149, Issue 5, Pages 492-495.

7. Gunay Gurleyik, Ali Aktekin, Fugen Aker,1 Hikmet Karagulle,2 and Abdullah Saglamc. Medical and Surgical Treatment of Idiopathic Granulomatous Lobular Mastitis: A Benign Inflammatory Disease Mimicking Invasive Carcinoma; J Breast Cancer. 2012 Mar;15(1):119-123.

8. Hee Ri Na Seo,1 Kuk Young Na,1 Hyun Ee Yim,2 Tae Hee Kim,3 Doo Kyoung Kang,3 Ki Keun Oh,3 Seok Yun Kang,4 Young-Sil An,5 Mison Chun,6 Woojae Kim,7 Rae Woong Park,7 Yong Sik Jung, 1 and $\mathrm{Ku}$ Sang Kim. Differential Diagnosis in Idiopathic Granulomatous 
Mastitis and Tuberculous Mastitis; J Breast Cancer. 2012 Mar;15(1):111-118.

9. Mimi Gangopadhyay, Anuradha De, Indranil Chakrabarti, Sailesh Ray. Idiopathic granulomatous mastitis-utility of fine needle aspiration cytology (FNAC) in preventing unnecessary surgery. Journal of the Turkish German Gynecology Association 11(3):127-30.

10. Leo FRANCIS Tauro,John Joseph S Martis, Celine George, Aroon Kamath. Tuberculous Mastitis Presenting as Breast Abscess; Oman Medical Journal 2011; 26(1):53-5.

11. Boakes E, Woods A, Johnson N, Kadoglou. Breast Infection: A Review of Diagnosis and Management Practices. Eur J Breast Health 2018; 14: 136-143.

12. Jorgensen MB, Nielsen DM. Diagnosis and treatment of granulomatous mastitis. Am J Med. 1992 Jul;93(1):97-101.

13. Lisa H. Amir, Della Forster, Helen McLachlan, Judith Lumley. Incidence of breast abscess in lactating women: report from an Australian cohort, BJOG: 2004; 111:1378-1381.

14. Foxman B, D'Arcy H, Gillespie B, Bobo JK, Schwartz K. Lactation mastitis: occurrence and medical management among 946 breastfeeding women in the United States. Am J Epidemiol 2002; 155: 103-114.

15. Jahanfar S, Ng CJ, Teng CL. Antibiotics for mastitis in breastfeeding women. Cochrane Database Syst Rev 2009: 1.

16. Kataria K, Srivastava A, Dhar A. Management of Lactational Mastitis and Breast Abscesses: Review of Current Knowledge and Practice. Indian J Surg 2013; 75: 430-435.

17. Branch-Elliman W, Golen TH, Gold HS, Yassa DS, Baldini LM, Wright SB. Risk factors for Staphylococcus aureus postpartum breast abscess. Clin Infect Dis 2012; 54: 71-77.
18. Rasha Mohamed Kamal, Soha Talaat Hamed. Classification of Inflammatory Breast Disorders and Step by Step Diagnosis; The Breast Journal 2009; 1-14.

19. Guray M, Sahin AA. Benign breast diseases: classification, diagnosis, and management. Oncologist. 2006 May;11 (5): 435-49.

20. S Thirumalaikumar, S Kommu, Aspiration of breast abscesses: Emerg Med J 2004; 21: 333-4.

21. Pontifex AH, Roberts FJ. Fine needle aspiration biopsy cytology in the diagnosis of inflammatory lesions. Acta Cytol. 1985 Nov-Dec;29(6):979-82

22. Monica Morrow, Evaluation of common breast problems;Am Fam Physician. 2000 Apr 15;61(8):2371-2378.

23. D. K. Das , P. Sodhani , V. Kashyap, S. Parkash , J. N. Pant, P. Bhatnagar. Inflammatory Lesions of the Breast: Diagnosis By Fine Needle: 1992: 3: 281-9. 